\title{
Endoscopic and histological pitfalls in the diagnosis of celiac disease: A multicentre study assessing the current practice
}

\author{
Mohammad Rostami-Nejad ${ }^{1,2}$, Vincenzo Villanacci ${ }^{3}$, Sabine Hogg-Kollars ${ }^{4}$, Umberto Volta ${ }^{5}$, Stefania Manenti ${ }^{3}$, \\ Mohammad Reza-Zali ${ }^{1}$, Giacomo Caio ${ }^{5}$, Paolo Giovenali ${ }^{6}$, Ausrine Barakauskiene ${ }^{7}$, Edita Kazenaite ${ }^{8}$, \\ Gabriel Becheanu9 ${ }^{9}$, Mircea Diculescu ${ }^{10}$, Salvatore Pellegrino ${ }^{11}$, Giuseppe Magazzù ${ }^{12}$, Giovanni Casella ${ }^{13}$, \\ Camillo Di-Bella ${ }^{14}$, Nicola Decarli ${ }^{15}$, Mauro Biancalani ${ }^{15}$, Gabrio Bassotti ${ }^{16}$ and Kamran Rostami ${ }^{17}$
}

\begin{abstract}
${ }^{I}$ Celiac Disease Department. Gastroenterology and Liver Diseases Research Center. Shahid Beheshti University of Medical Sciences. Tehran, Iran. ${ }^{2}$ Department of Gastroenterology. VU University Medical Centre. Amsterdam, Netherlands. ${ }^{3}$ Department of Pathology Spedali Civili. Brescia, Italy. ${ }^{4}$ School of Immunity \& Infection. University of Birmingham. United Kingdom. ${ }^{5}$ Department of Gastroenterology and Internal Medicine. St Orsola-Malpighi Hospital. University of Bologna. Bologna, Italy. ${ }^{6}$ Diagnostic Cytology and Histology Unit. Ospedale Santa Maria della Misericordia. Perugia, Italy. ${ }^{7}$ Vilnius University. Medical Faculty. National Center of Pathology. Lithuania. ${ }^{8}$ Vilnius University. Medical Faculty. Lithuania. ${ }^{9}$ Senior Lecturer Carol Davila University of Medicine and Pharmacy. Department of Pathology. Bucharest, Romania. ${ }^{10}$ Fundeni Clinical Institute. Clinical of Gastroenterology and Hepatology. Bucharest,

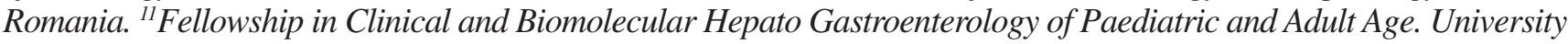
Hospital "G. Martino". Messina, Italy. Regional Celiac Center. ${ }^{12}$ Regional Celiac Center. University Hospital "G. Martino”. Messina. Italy. ${ }^{13}$ Medical Department. Desio Hospital Desio (Monza e Brianza). Italy. ${ }^{14}$ Department of Pathology. Desio Hospital Desio (Monza e Brianza). Italy. ${ }^{15}$ Department of Diagnostic-Unit of Pathology "San Giuseppe Hospital” (USL11) Empoli Florence, Italy. ${ }^{16}$ Gastroenterology and Hepatology Section. Department of Clinical and Experimental Medicine. University of Perugia. Italy. ${ }^{17}$ Department of Gastroenterology. Luton \& Dunstable Hospital NHS Foundation Trust. Luton,United Kingdom
\end{abstract}

\begin{abstract}
Background and aims: the diagnosis of celiac disease requires small bowel biopsies to identify the characteristic mucosal changes. The current biopsy practice among endoscopists for celiac disease is in most part unknown. The aim of this study was to compare the different diagnostic policies in various centers in their current practice.

Method: information from a total of 931 confirmed celiac disease patients was retrospectively obtained retrospectively from nine centers in European and Middle Eastern countries. The number of small-bowel biopsies obtained from the duodenal bulb and the second part of the duodenum was compared among different centers.
\end{abstract}

Results: the most frequent stage of mucosal changes amongst Iranian subjects was Marsh IIIa whereas in the rest of the study population was Marsh IIIc. Marsh I and Marsh II were more prevalent in adults $(P<0.05)$ and Marsh IIIc was significantly higher in pediatric ages between 1 and $15(P<0.05)$. The most common number of biopsy specimens obtained from Romanian subjects was 1 (52\% of cases), followed by 2 for Iranian (56\%), 3 for Lithuanian $(66.7 \%)$ and British patients (65\%) and 4 for Italian patients (48.3\%). For majority of cases, anemia was the most prevalent symptom (18.7\%) followed by malabsorption (10.5\%), diarrhea $(9.3 \%)$ and dyspepsia (8.2\%), respectively.

Conclusions: despite the evidence-based recommendations, this study revealed a poor compliance with major guidelines on diagnosis of celiac disease. We emphasize that taking adequate number of duodenal biopsies should be implemented for an accurate diagnosis and also for the exclusion of celiac disease.

Key words: Celiac disease. Pitfall. Biopsy specimen. Multicenter.

Rostami-Nejad M, Villanacci V, Hogg-Kollars S, Volta U, Manent S, Reza-Zali M, Caio G, Giovenali P, Barakauskiene A, Kazenaite E, Becheanu G, Diculescu M, Pellegrino S, Magazzù G, Casella G, Di-Bella C, Decarli N, Biancalani M, Bassotti G, Rostami K. Endoscopic and histological pitfalls in the diagnosis of celiac disease: $A$ multicentre study assessing the current practice. Rev Esp Enferm Dig 2013;105:326-333
Correspondence: Kamran Rostami. Department of Gastroenterology, Luton \& Dunstable Hospital NHS Foundation Trust. Luton Lewsey Road, Luton LU4 ODZ, United Kingdom.

e-mail: kamran.rostami@nhs.net

$\ldots$




\section{INTRODUCTION}

Diagnosis of celiac disease (CD) is one of the most challenging aspects of this condition. The immune response to the grains of wheat, rye, and barley leads to progressive damages in the small intestine, affecting differently many individuals and resulting in the production of $\operatorname{IgA}$ serum antibodies directed against tissue transgutaminase (tTG) in most but not all (1-4).

Despite the availability of specific and sensitive serological tests, diagnosis of atypical forms of CD could be very challenging (5). Intestinal biopsy is still considered an essential diagnostic tool for detection of CD in different stages (6). Histology also permits to evaluate the response to a gluten-free diet, should there be any doubt about the diagnosis.

The sensitivity of the serological tests available might be correlated with the degree of mucosal abnormalities and according to many studies, a negative serological test may not always exclude the diagnosis of CD (7). The mucosal lesions in CD can be patchy, and if the sampling is insufficient, the risk of missing the diagnosis increases $(8,9)$. Low degrees of mucosal abnormalities like microscopic enteritis in small intestinal specimens are not specific for $\mathrm{CD}$, but a combination of clinical, serological and genetic evaluation may help to confirm the diagnosis (10). If possible, it would be more desirable to avoid endoscopy completely. We might argue that endoscopy may be unnecessary a small subgroup of patients who have classic symptoms and high level positive serology, but we still find that taking a biopsy appears essential in the majority of cases (11). A multicentre endoscopy database study found that most patients undergoing upper GI endoscopy for indications such as anemia, iron deficiency, and weight loss had no duodenal biopsy taken during the procedures (12-14). Moreover, a normal endoscopic appearance lacks sufficient sensitivity to exclude CD $(15,16)$.

As mucosal abnormalities in $\mathrm{CD}$ are patchy and the orientation of biopsies are variable, multiple biopsies (4-6 biopsies) from the duodenal bulb and descending duodenum are usually recommended as standard method for CD evaluation (10,17-19). Study results by Pais and coworkers showed that 2 biopsies confirmed CD in $90 \%$ of patients, while 4 biopsies established a diagnosis in $100 \%$ of cases (10). By using a large, international, pathology database, we assessed the adherence to these proposed guidelines.

In this study, we investigated the procedure-related factors associated with the submission of biopsy specimens and compared the different diagnostic criteria in various centers in Italy, Iran, Lithuania, Romania and the UK respectively. This study elucidates how a guideline is exercised in clinical practice, both in terms of adherence to the recommendation as well as the incremental yield of adherence.

\section{MATERIAL AND METHODS}

\section{Patients and settings}

In this retrospective multicentre study, data were collected in the period May 2009-May 2011 from Iran (Tehran), Romania (Bucharest), Lithuania (Vilnius), the United Kingdom (Birmingham) and 6 different areas in Italy, including Brescia, Bologna, Desio, Messina, Perugia and Empoli. For each center, at least 100 confirmed CD patients with endoscopic procedures and small bowel biopsies were included.

\section{Diagnosis of celiac disease in different centers}

Histopathological findings in all centers were evaluated in accordance with the modified Marsh classification (1). The final diagnosis of celiac disease was based on a combination of clinical, analytical and histological data, and a response to a gluten-free diet, and defined by clinicians in charge of clinical care of patients.

In Iran, IgA-tTG antibody and immunoglobulin A were measured using a commercially available kit (AESKULISA tTG, Germany) and an enzyme linked immunosorbent assay (ELISA) method. In Italy, IgA-anti-endomysial antibodies (EMA) were investigated by indirect immunefluorescence that used human umbilical cord cryostat sections. Sera were tested at the initial dilution of 1:5 and when positive, titrated up to the end point. In Romania, the patients suspected for $\mathrm{CD}$ were usually referred directly for the gastroenteroscopy because of lack of funding for human leukocyte antigen (HLA)-typing and serology is performed only in cases with suspected CD at biopsy.

The duodenal specimens in Bucharest were oriented using Endokit and the biopsies in some departments in Italy (Brescia, Desio and Messina) were oriented on acetate cellulose filters.

\section{Duodenal biopsy and histology classification}

To determine the number of duodenal biopsy specimens for each biopsy set, we used a free-text search of the pathologist's description of each sample. When present, specimens from the duodenal bulb were included in the total number of specimens submitted. In each lab, biopsies were interpreted by expert gastrointestinal pathologists (blinded to the clinical data). Centers with the lowest and highest number of biopsy specimens were analyzed separately and also compared in order to investigate causes for those differences.

All centers used the cutoff of $>25$ intraepithelial lymphocytes per 100 enterocytes for a Marsh I stage, increased intraepithelial lymphocytes accompanied by crypt hyperplasia (Marsh II), partial (Marsh 3A), subtotal (Marsh 3B) 
and total villous atrophy (Marsh 3C) as described in modified classification (1). According to the follow up reports to ordering HLA typing, the genetic data available were incomplete and HLA DQ typing was carried out only in some centers.

\section{Data collection form}

During the same period, the number of biopsy sample taken from each center and their clinical data were compared. For each specimen, the following information was available: sex and age of the patient, location and sample provider, summary of the clinical history, serology/genetic and histopathological findings.

GI symptoms such as abdominal pain, diarrhea, constipation, nausea and vomiting, weight loss and flatulence as well as additional signs and symptoms such as iron deficiency anemia, osteoporosis, hypertransaminasemia, malabsorption, and other related abnormalities were recorded. Malabsorption was defined by a decreased body mass index, weight loss and low triglyceride, cholesterol, ferritin or serum albumin and also anemia was defined when the level of hemoglobin and hematocrit (HCT) was decreased less than the normal quantity.

\section{Statistical analysis}

We used the chi-square test to assess the association between adherence to the recommendation of submitting $\geq 4$ specimens and the proportion of patients with pathological findings consistent with $\mathrm{CD}$. The results with a p-value $<0.05$ were considered statistically significant. Because our data set did not contain information regarding HLA typing for all cases and clinical follow-ups, we defined a priority that results with either blunted villi (Marsh 3A) or flat villi (Marsh 3B/C) were meeting the pathological definition of $\mathrm{CD}$. For assessing the relationship between ordinal categories such as the number of specimens and the pathologic diagnosis of CD, we used the Fisher's Exact Test for trend.

\section{RESULTS}

Overall, 658 women and 273 men (mean age at diagnosis $35.4 \pm 17.25$ years, median age 36 years, age range 1-72 years) with a diagnosis of $C D$ were included. Patients ranged 1-15 year-old were considered as children and older as adults. In all participating countries females were predominant ( $70.7 \%$ of patients), and the mean age ( \pm standard deviation [SD]) was $36.6 \pm 16.4$ years. Lithuanian patients were the oldest, with a mean age of $43.7 \pm 18.6 \mathrm{yrs}$, and Iranian patients the youngest $(30 \pm 13.6 \mathrm{yrs})$. There was a statistically significant differences between the genders in studied countries $(p=0.0001)$. Fifty one patients were excluded based on their incomplete data collection.

Table I showed demographical, clinical, and histopathological data in the study population.

One hundred and four samples $(11.17 \%)$ were collected from the bulb and the rest was collected from the second duodenal portion. Marsh I and II lesions were noted in 322 individuals (34.5\%), Marsh 3A was found in 180 (19.3\%), Marsh 3B in 178 (19.1\%) and Marsh 3C in 233 study subjects $(25 \%)$ (Fig. 1). When a pathological diagnosis of $\mathrm{CD}$ was defined as that of blunted or flat villi (Marsh 3A/B/C), a total of 591 individuals $(63.4 \%)$ were categorized as having $\mathrm{CD}$. According to the Marsh classification there were statistically significant differences among studied countries $(p=0.0001)$. A Marsh 0 stage was recorded in 18 individuals $(2 \%)$ being most of them were under 15 years old.

According to age groups Marsh IIIc was significantly more prevalent in pediatric patients (aged 1-15) compared to other age groups $(p=0.0001)$ and Marsh I and II were predominantly present in adult patients $(p=0.0001)$.

Regarding the number of biopsy specimens taken per CD case, we found that 1 biopsy specimen had led to a CD diagnosis in $63(6.7 \%)$ cases (11 specimens were Marsh I and II and the rest was higher stages), 2 in 212 (22.7\%) cases (96 specimens were Marsh II or lower), 3 in 306 $(32.9 \%)$ cases (153 were Marsh II or lower), 4 in 299 (32.1\%) cases (81 specimens were Marsh II or lower) and $\geq 5$ in $51(5.5 \%)$ cases ( 9 specimens were Marsh II or lower). The average number of biopsy specimens per patient was 3 (Table II, Fig. 2). The number of available biopsy samples directly associated with increasing Marsh stages $(p=0.0001)$.

The most common number of biopsy specimens taken from Romanian subjects was $1(52 \%)$ followed by 2 from Iranian patients $(56 \%), 3$ from Lithuanian $(66.7 \%)$ and British patients (65\%) and 4 from Italian patients $(48.3 \%)$. Villous atrophy (partial, subtotal or total) was the predominant histological lesion in all studied countries (Fig. 1). There was a statistically significant differences regarding number of biopsy specimens taken from studied counties $(p=0.0001)$.

The most common number of biopsy specimens taken from patients age 1-15 years old ( 83 cases) and adults age 31-45 (117 cases) was 3 . This differences was statistically significant for Italian and Lithuanian patients $(p=0.0001)$.

The most frequent degree of villous atrophy for Iranian subjects was $3 \mathrm{~A}$ and for the rest of the study population $3 \mathrm{C}$ (Table III). According to the histological variability illustrated above, a statistical correlation was found between type and distribution of the histological lesions and the number of biopsy specimens $(p=0.0001)$. Four or more duodenal biopsies had been taken for $58.7 \%$ of the Italian, $37 \%$ of the British, $7 \%$ of the Romanian and $5 \%$ of the Iranian studied subjects. None of the Lithuanian patients had 4 or more duodenal biopsies. 
Table I. Demographic, clinical and histopathological information in the study population

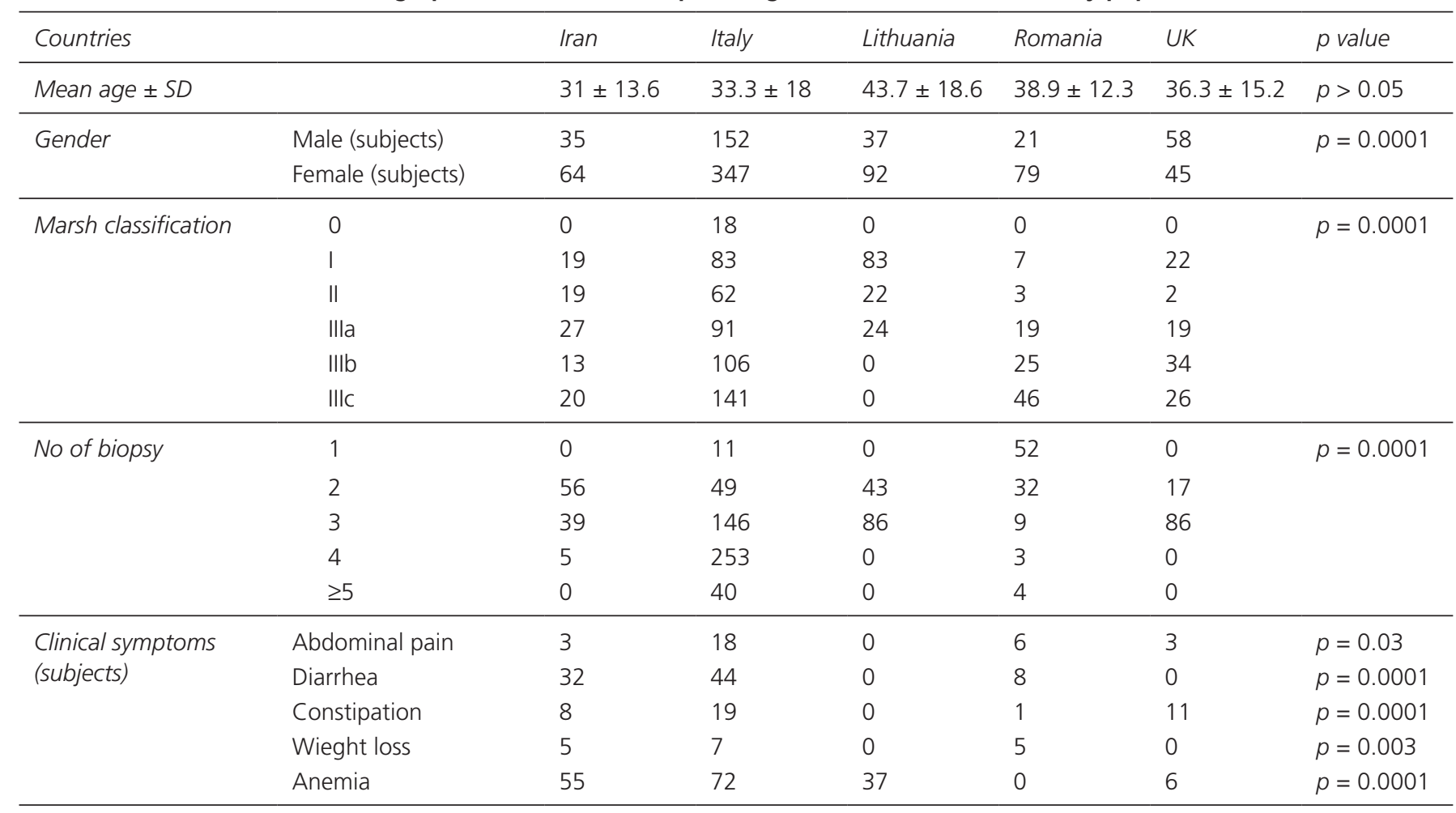

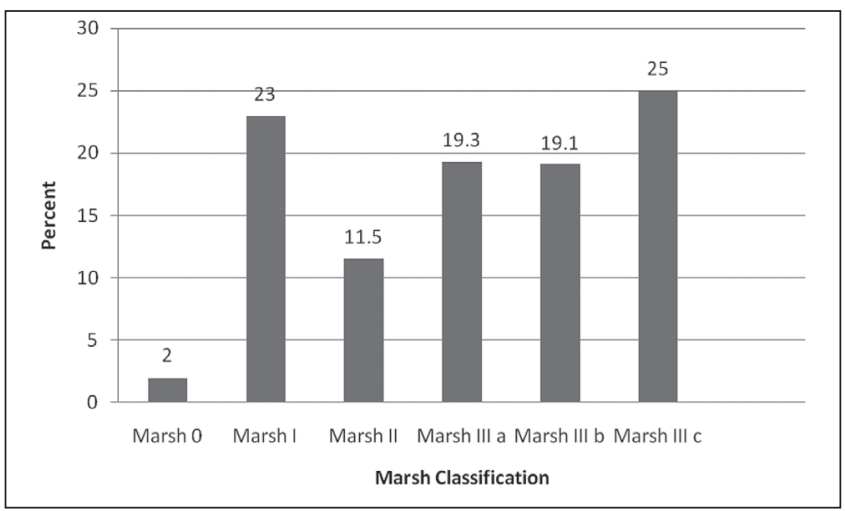

Fig. 1. Distribution of mucosal changes in our study group according to modified Marsh classification for celiac disease.

Routine immune-histochemical evaluation of T lymphocytes with CD3 and CD8 antibodies was used only in Iran and one center in Italy (Brescia).

To confirm the diagnosis of celiac disease, serological assessments were performed in all the participating centers.

Anti-EMA resulted positive in $32.3 \%$ of Iranian patients; positive anti-tTG IgA was documented on $67.7 \%$ on Italians, and positive anti gliadine IgA antibodies were documented in $6.3 \%$ of the UK patients. Regarding to Lithuanian patients $34 \%$ of them exhibited positive anti

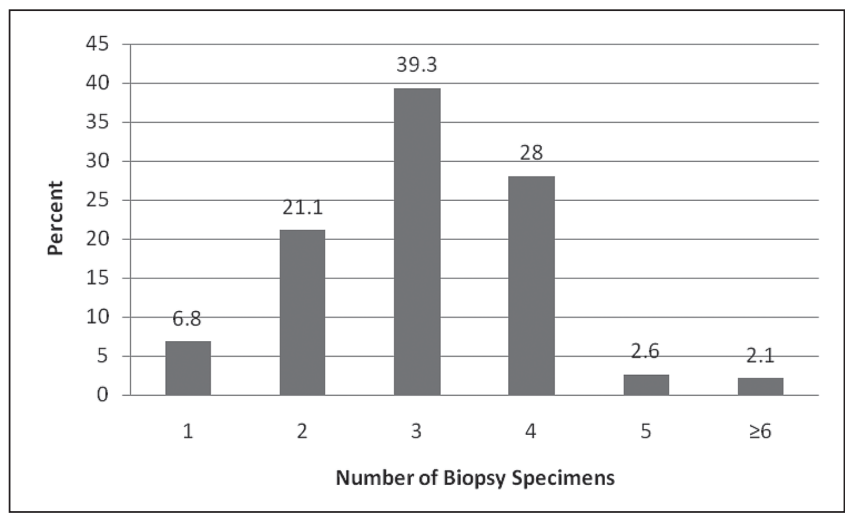

Fig. 2. The percent of specimens submitted for the diagnosis of celiac disease.

gliadine $\operatorname{IgA}$, being the remaining positive for anti-tTG IgA antibodies.

HLA typing was not available for Lithuanian subjects, and if performed, there was no access to genetic results of the HLA DQ typing in this country. HLA typing was performed for very few selected cases in Romania. HLA-typing for Italian (72/602) and Iranian subjects (52/100) showed that, HLA DQ2 was the predominant genotype for both countries.

The clinical data varied from one region to the other; however, anemia was the main presenting symptom 
Table II. Frequency of different stages of mucosal abnormalities and correlation with the number of duodenal biopsy samples obtained

\begin{tabular}{|c|c|c|c|c|c|c|}
\hline N. ${ }^{\circ}$ of biopsy & \multicolumn{6}{|c|}{ Modified Marsh classification } \\
\hline 1 & 0 & 10 & 1 & 15 & 15 & 22 \\
\hline 2 & 1 & 69 & 23 & 31 & 28 & 45 \\
\hline 3 & 15 & 104 & 38 & 69 & 45 & 95 \\
\hline$\geq 5$ & 0 & 3 & 4 & 3 & 8 & 26 \\
\hline
\end{tabular}

CHP: crypt hyperplasia; EC: enterocytes; IEL: intraepithelial lymphocytes; PVA: partial villous atrophy; STVA: subtotal villous atrophy; TVA: total villous atrophy (Ref. 1). There was a statistically significant association between number of biopsy specimens and stages of Marsh classification $(p=0.0001)$.

\section{Table III. Frequency of the different degrees of mucosal abnormalities based on modified Marsh classification in different countries}

\begin{tabular}{|c|c|c|c|c|c|c|c|c|}
\hline \multirow[t]{2}{*}{ Country } & \multirow{2}{*}{$\begin{array}{l}N .^{\circ} \text { of } \\
\text { biopsy }\end{array}$} & \multicolumn{6}{|c|}{ Modified Marsh classification } & \multirow[t]{2}{*}{ Total } \\
\hline & & 0 & 1 & 2 & $3 \mathrm{~A}$ & $3 B$ & $3 C$ & \\
\hline \multirow{3}{*}{ Iran } & 2 & 0 & 13 & 10 & 12 & 9 & 11 & 55 \\
\hline & 3 & 0 & 6 & 7 & 13 & 4 & 8 & 38 \\
\hline & 4 & 0 & 0 & 2 & 2 & 0 & 1 & 5 \\
\hline \multirow[t]{5}{*}{ Italy } & 1 & 1 & 7 & 0 & 3 & 0 & 1 & 12 \\
\hline & 2 & 1 & 17 & 2 & 13 & 6 & 11 & 50 \\
\hline & 3 & 14 & 29 & 16 & 14 & 13 & 60 & 146 \\
\hline & 4 & 2 & 27 & 40 & 59 & 81 & 44 & 253 \\
\hline & $\geq 5$ & 0 & 3 & 4 & 2 & 6 & 25 & 40 \\
\hline \multirow[t]{2}{*}{ Lithuania } & 2 & 0 & 32 & 10 & 0 & 0 & 1 & 43 \\
\hline & 3 & 0 & 51 & 12 & 0 & 0 & 23 & 86 \\
\hline \multirow[t]{5}{*}{ Romania } & 1 & 0 & 3 & 1 & 12 & 15 & 21 & 52 \\
\hline & 2 & 0 & 2 & 1 & 4 & 6 & 19 & 32 \\
\hline & 3 & 0 & 1 & 1 & 1 & 1 & 5 & 9 \\
\hline & 4 & 0 & 1 & 0 & 1 & 1 & 0 & 3 \\
\hline & $\geq 5$ & 0 & 0 & 0 & 1 & 2 & 1 & 4 \\
\hline \multirow{2}{*}{$\begin{array}{l}\text { United } \\
\text { Kingdom }\end{array}$} & 2 & 0 & 5 & 0 & 1 & 7 & 4 & 17 \\
\hline & 3 & 0 & 17 & 2 & 18 & 27 & 22 & 86 \\
\hline
\end{tabular}

in $174(18.7 \%)$ patients, malabsorption appeared in $98(10.5 \%)$, diarrhea in $87(9.3 \%)$ and dyspepsia in 77 (8.2\%) patients, respectively (Fig. 3). There was a statistically significant correlation between these symptoms and abnormality in histology $(p<0.05)$. As shown in figure 3 , anemia and diarrhea associated with Marsh 3c stage, malabsorption was predominant in Marsh 1, and dyspepsia associated with Marsh 3b. Ninety percent of the Lithuanian study subjects were affected by malabsorption. The most frequent symptom in Italian patients was dyspepsia
(73 cases [14.6\%], probably related with a high-carbohydrate diet), followed by anemia (71 cases [14.2\%]) and diarrhea (44 cases [8.8\%]). For Iranian subjects the predominant symptoms were anemia (55 cases [55\%]), diarrhea (32 cases [32\%]) and osteopenia (25 cases [25\%]). Romanian subjects predominantly complained of diarrhea (8 cases [8\%]), abdominal pain (6 cases [6\%]) and weight loss (5 cases [5\%]). Statistically significant differences were observed when comparing the reported clinical symptoms in different countries $(p<0.05)$.

Abdominal pain and failure to thrive were significantly reported in pediatrics $(p=0.01)$ and anemia, osteoporosis, osteopenia and dyspepsia were more prevalent in adults $(p<0.05)$ (Table IV).

In spite of the histological variability illustrated in this study, a significant correlation was found between type and distribution of the histological abnormalities and some of the clinical symptoms such as abdominal pain, diarrhea, low vitamin B12/Acid folic serum levels, malabsorption, IgA deficiency, dyspepsia, asthenia and failure to thrive $(p<0.05)$.

Among the 931 study subjects, 419 (45\%) displayed typical presentation, $430(46.2 \%)$ atypical presentation,

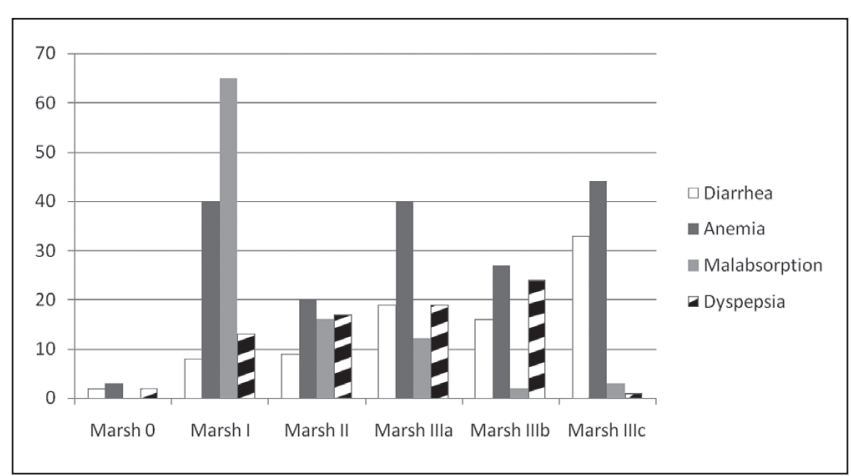

Fig. 3. Comparison of rates of anemia, diarrhea, dyspepsia and malabsorption in cases of celiac disease with abnormal histology (Marsh $0-\|$ and Marsh IIlabc). 
Table IV. Different signs and symptoms in pediatric compare to adult celiac patients retrospectively recruited in our multicenter study

\begin{tabular}{llll}
\hline Sign and symptoms & Pediatrics (\%) $n=127$ & Adults (\%) $n=804$ & $p$ value \\
\hline Abdominal pain & $11(8.7)$ & $19(2.3)$ & 0.01 \\
Diarrhea & $9(7)$ & $75(9.3)$ & 0.3 \\
Constipation & $7(5.5)$ & $32(4)$ & 0.7 \\
Anemia & $24(18.9)$ & $145(18)$ & 0.03 \\
Osteoporosis & 0 & $13(1.6)$ & 0.003 \\
Osteopenia & 0 & $30(3.7)$ & 0.005 \\
Hypertransaminasemia & 0 & $19(2.3)$ & 0.01 \\
Weight loss & $4(3.14)$ & $75(1.6)$ & 0.83 \\
Dyspepsia & $1(0.78)$ & $12(1.5)$ & 0.001 \\
Bloating & $1(0.78)$ & $5(0.62)$ & 0.48 \\
Failure to thrive & $15(11.8)$ & & 0.0001 \\
\hline
\end{tabular}

and $64(6.9 \%)$ had no or only minor GI symptoms. A statistically significant correlation was found between abnormal histology and abdominal pain, dyspepsia and failure to thrive for Italian patients and between abnormal histology and anemia for Lithuanian patients $(p<0.05)$.

\section{DISCUSSION}

Even though noninvasive antibody testing is an excellent screening tool for CD, endoscopic evaluation via duodenal biopsy in suspected patients remain crucial in the diagnosis of clinical atypical CD.

The typical symptoms associated to CD are abdominal pain, chronic diarrhea, vomiting, weight loss, foul smelling stool, fatigue, failure to thrive or short stature, delayed puberty, osteoporosis and anemia, and the atypical celiac disease symptoms include obesity, dyspepsia, constipation, depression and anxiety (4).

The number of diagnosed patients has increased; this may be due to a greater proportion of outpatients being investigated for relatively minor symptoms, especially elderly people who visit their physicians more often (20).

In this analysis of an international pathology database of duodenal biopsies, only $37.6 \%$ of patients had $\geq 4$ specimens submitted upon upper endoscopy. Adherence to the proposed standard remained low even for patients with typical CD, with fewer than $40 \%$ of such patients having $\geq 4$ specimens submitted $(1,17,20,21)$. Italy had the best record of adequate sampling in this study and the rest of participant disappointingly failed to adhere to evidence based recommendations.

Biopsy handling and experience of pathologists in different sites including duodenal bulb (19) was also variable in this study. It is suggested to take multiple biopsies from different duodenal sites in all cases where CD is suspected in order to minimize the risk of misdiagnosis
(10). Differences in practice are highlighted and we can see the different approaches and diagnostic workups in countries participated in this evaluation. Biopsy specimens taken from the descending duodenum as well as jejunal specimens produced good results. However, duodenal bulb biopsy specimens despite being very important $(17,19)$, were poorly taken in this study. In addition, a duodenal biopsy should have a reasonable size, be well orientated and adequately stained and should be thoroughly examined by an experienced GI pathologist (22). There was not a reliable tool available to assess all these qualities in the biopsy samples because of the nature of this multicentre study. Although general pathologists might not be aware of the variety of pathological changes seen in CD, in a positive note all the pathologists participated in this study had expertise and interest in histology of CD.

Villanacci et al. suggested that the biopsy taken from anywhere along the duodenum could theoretically lead to the diagnosis of CD if the specimen was correctly obtained and assessed according to the standards $(22,23)$.

In a recent review, Yantiss \& Odze (2009) referred to the "optimal method of obtaining biopsies in patients with celiac disease" as "controversial" (24). It may be stressed at this point that the proposed guideline entirely derives from the observation that the histopathologic abnormalities of CD are patchy, and can be missed if an insufficient quantity of specimens is submitted. The recommendation is supported by a single-centre retrospective study of 93 patients with $\mathrm{CD}$, which found that 4 specimens led to a positive diagnosis in $100 \%$ of patients, whereas 2 specimens were diagnostic in only $90 \%$ of patients (10). The authors concluded that at least 4 duodenal biopsy specimens should be taken to rule out CD. A second study, investigating 56 patients with known $\mathrm{CD}$, found that 3 biopsy specimens were sufficient as long as 1 specimen was obtained from the duodenal bulb; however, 5 biopsy specimens were necessary to recognize the most severe 
extent of villous atrophy. These studies are limited by small sample size and single-center settings (25). Evans et al. recently solve these problems (19). They investigated overall 461 patients including 126 newly diagnosed CD, 85 established CD, and 250 controls. Their study suggests that the optimal strategy for diagnosing CD in both suspected patients with positive serology and those with established CD could only achieve $100 \%$ sensitivity by always incorporating a duodenal bulb biopsy (19).

In this study, taking 1-2 biopsy specimens led to the confirmation of diagnosis in $29.5 \%$ of cases, while more than 3 biopsy specimens led to the diagnosis in $70.5 \%$. We found a statistical correlation $(p<0.05)$ between the number of biopsies and histological abnormalities. The variation in the number of biopsy samples in this study may be associated with the endoscopists' practice in each region. Endoscopists in Romania, Iran and Lithuania are more likely to perform less and fewer numbers of biopsies compared to endoscopists in Italy.

Because villous atrophy in CD is patchy and the orientation of the specimens is variable, 4 to 6 random biopsy specimens including at least one from the duodenal bulb are recommended in order to maximize the sensitivity and achieve a correct diagnosis of CD $(10,17,19,22)$. This may suggest that if additional biopsy specimens in our study had been taken from subjects CD may have been confirmed in more cases (10).

The incremental yield varied with indication and was greatest when the symptom was anemia (18.7\%). However, submitting $\geq 4$ specimens also increased the diagnostic yield of CD even when the indication was dyspepsia $(8.2 \%)$. As $H$. pylori infection is associated with some mucosal abnormalities in dyspeptic patients, Lecleire et al. suggest that endoscopic markers of villous atrophy are not useful for the detection of CD in patients with dyspeptic symptoms (26).

Retrospective analysis of pathology tissue databases produced high-quality analyses of GI epidemiology and quality measures $(27,28)$. In our study, conducted in a similar manner, we have some limitations. For instance, we did not have access to socioeconomic or racial data to determine whether these individual patient characteristics were associated with the submission of the recommended number of specimens. We also had no access to key variables that influence the likelihood of CD, such as data regarding family history of $\mathrm{CD}$. We looked for including patients with confirmed diagnosis for most of the locations. We did not describe a yield of biopsies because the total number of patients biopsied for suspected CD and control group was not included. Therefore, the false negative rate was not assessed.

Moreover, information regarding the type of sedation used during the procedure and the degree of sedation, which may have impacted the ability to obtain $\geq 4$ specimens, was not available. The degrees to which endoscopists adhere to such recommendations in clinical practice and the diagnostic yield of adherence to this standard have not been studied.

Since the study was retrospective and many patients did not have uniform information regarding the degree of adherence to the gluten-free diet in their medical record, we were not able to assess the correlation between seronegativity after treatment and the degree of compliance to the GFD (27). For example in Bucharest, for the majority of the patients that are suspected to have CD, endoscopy is performed prior to serological. After the histological diagnosis compatible with $\mathrm{CD}$, patients tend to have serological tests and, rarely, genetic testing. As methodological approach to the diagnosis of $\mathrm{CD}$ in this region may be attributed to high costs involved with genetic testing for DQ2/DQ8 (more than minimum salary/month), HLA typing is rarely performed. A similar situation is present in Iran and Lithuania; because HLA typing is expensive and access to laboratories that can perform genetic testing is limited, HLA typing is rarely carried out, and if performed, then it will be only in specific cases. This may represent the main obstacle for clinicians working without the facility for HLA-typing. However, the situation is not better in UK, as HLA typing is not popular between clinician again due to high cost and National Health Service strict cost saving policies. According to the current literature serology only at high level $>10 \mathrm{x}$ above normal value and histology only with Marsh IIIb-c have the highest sensitivity and specificity close to $100 \%$ for CD. HLA would be very useful in excluding the diagnosis thanks to its very high negative predictive value.

Another obstacle for correct diagnosis in all investigated geographical locations may be that clinicians generally do not share clinical data or serology results of patients with pathologists. Most pathologists ignore the subtle histological changes like microscopic enteritis (29) in absence of such clinical data. In our opinion, this practice indicates the different levels of approach involved with the correct histological diagnosis of CD in different centers. Pathologists in general should know the subtle manifestations and presentations of the disease. The evidence suggests that sharing the clinical data with pathologists might be essential toward a correct diagnosis as the pathologist is able to detect the majority of CD patients (28). Despite the existent evidence, counting of IEL is still not a routine practice in many West European countries. 'Clearly this is the first step in identifying explanation for many labeled as Functional Gut Disorders'.

Regarding to clinical presentation of $\mathrm{CD}$, our results indicate that a classical form of celiac disease is predominant in pediatric patients compared to adult $\mathrm{CD}$, and greater severity and intestinal abnormality are sawn in younger patients. In contrast, atypical forms predominate among adults, with lower stages of duodenal damage. Our results are compatible with those by Rodrigo-Sáez et al. (30).

As a conclusion, we should implement the biopsy guidelines with $\geq 4$ biopsy specimens into clinical practice 
including or with additional samples from bulb (17). In our multicentre study, only the minority of patients with upper GI endoscopy and duodenal biopsy except Italian cases had $\geq 4$ specimens. In the light of the beneficial effect on the histological diagnosis of CD (31), in particular in atypical cases increased adherence to biopsy guidelines is warranted. In typical cases, as suggested by European Society for Pediatric Gastroenterology and Nutrition (ESPGAN), the endoscopy and biopsy obviously might be avoided. However, the majority of celiac patients present with atypical manifestation where endoscopy and the correct sampling approach are paramount in diagnostic pathway (5).

\section{ACKNOWLEDGEMENT}

We would like to thank Dr R. Singh, Pathologist from Good Hope Hospital, Birmingham. The Romanian data collection was supported by the Sectoral Operational Programme Human Resources Development, financed from the European Social Fund and by the Romanian Government under the contract number POSDRU/89/1.5/S/64153. This paper is resulted from $\mathrm{PhD}$ thesis of Mohammad Rostami Nejad.

\section{REFERENCES}

1. Rostami K, Kerckhaert J, Tiemessen R, Von Blomberg BM, Meijer JW, Mulder CJ. Sensitivity of antiendomysium and antigliadin antibodies in untreated Coeliac disease: disappointing in clinical practice. Am J Gastroenterol 1999;94:888-94.

2. Rostami-Nejad M, Villanacci V, Mashayakhi R, Molaei M, Bassotti $\mathrm{G}$, Zojaji $\mathrm{H}$, et al. Celiac disease and Hp infection association in Iran. Rev Esp Enferm Dig 2009;101:850-4.

3. Ravelli A, Bolognini S, Gambarotti M, Villanacci V. Variability of histologic lesions in relation to biopsy site in gluten-sensitive enteropathy. Am J Gastroenterol 2005;100:177-86.

4. Rostami Nejad M, Rostami K, Pourhoseingholi MA, Nazemalhosseini Mojarad E, Dabiri H, Habibi M, et al. Atypical presentation is dominant and typical for coeliac disease. J Gastrointestin Liver Dis 2009;18:285-91.

5. Ribes-Koninckx C, Mearin M, Korponay-Szabó I, Shamir R, Husby S, Ventura A, et al. Coeliac Disease Diagnosis: ESPGHAN 1990 Criteria or Need For a Change? Results of a Questionnaire. J Pediatr Gastroenterol Nutr 2012:54:15-9.

6. Cammarota G, Cuoco L, Cesaro P, Santoro L, Cazzato A, Montalto M, et al. A highly accurate method for monitoring histological recovery in patients with celiac disease on a gluten-free diet using an endoscopic approach that avoids the need for biopsy: a double-center study. Endoscopy 2007;39:46-51.

7. Abrams JA, Diamond B, Rotterdam H, Green PH. Seronegative celiac disease: increased prevalence with lesser degrees of villous atrophy. Dig Dis Sci 2004;49:546-50.

8. Bonamico M, Mariani P, Thanasi E, Ferri M, Nenna R, Tiberti C, et al Patchy villous atrophy of the duodenum in childhood celiac disease. $\mathrm{J}$ Pediatr Gastroenterol Nutr 2004:38:204-7.

9. Vogelsang H, Hänel S, Steiner B, Oberhuber G. Diagnostic duodenal bulb biopsy in celiac disease. Endoscopy 2001;33:336-40.
10. Pais WP, Duerksen DR, Pettigrew NM, Bernstein CN. How many duodenal biopsy specimens are required to make a diagnosis of celiac disease? Gastrointest Endosc 2008;67:1082-7.

11. Rostami K, Danciu M. Endoscopy and small-bowel biopsy in celiac disease: indications and implications. Endoscopy 2007;39:46-51.

12. Harewood GC, Holub JL, Lieberman DA. Variation in small bowel biopsy performance among diverse endoscopy settings: results from a national endoscopic database. Am J Gastroenterol 2004;99:1790-4.

13. Ishaq S, Mahmood R, Vilannacci V, Bassotti G, Rostami K. Avoiding biopsy in iron deficiency anemia is not a cost-effective approach. Rey Esp Enferm Dig 2012;104:334-5.

14. Sáez LR, Álvarez DF, Martínez IP, Mieres NA, García PN, García Rde F, et al. Refractory iron-deficiency anemia and gluten intolerance. Response to gluten-free diet. Rev Esp Enferm Dig 2011;103:349-54.

15. Smith AD, Ramesar K, Dunk AA. Routine duodenal biopsies to exclude celiac disease? Not yet. Gastrointest Endosc 2004;60:164-5.

16. Weir DC, Glickman JN, Roiff T, Valim C, Leichtner AM. Variability of histopathological changes in childhood celiac disease. Am J Gastroenterol 2010;105:207-12.

17. Eloubeidi MA, Vilmann P, Wiersema MJ. Endoscopic ultrasound-guided fine-needle aspiration of celiac lymph nodes. Endoscopy 2004;36:901-8.

18. Hopper AD, Sanders DS. Obtaining duodenal biopsy specimens for celiac disease: is site as important as number? Gastrointest Endosc 2009;69:389-90.

19. Evans KE, Aziz I, Cross SS, Sahota GR, Hopper AD, Hadjivassiliou M, et al. A prospective study of duodenal bulb biopsy in newly diagnosed and established adult celiac disease. Am J Gastroenterol 2011;106:1837-742.

20. Collin P, Huhtala H, Virta L, Kekkonen L, Reunala T. Diagnosis of celiac disease in clinical practice: physician's alertness to the condition essential. J Clin Gastroenterol 2007:41:152-6.

21. Rostom A, Murray JA, Kagnoff MF. American Gastroenterological Association (AGA) Institute technical review on the diagnosis and management of celiac disease. Gastroenterology 2006;131:1981-2002.

22. Ravelli A, Villanacci V, Monfredini C, Martinazzi S, Grassi V, Manenti S. How patchy is patchy villous atrophy? Distribution pattern of histological lesions in the duodenum of children with celiac disease. Am J Gastroenterol 2010;105:2103-10.

23. Freeman HJ. Pearls and pitfalls in the diagnosis of adult celiac disease Can J Gastroenterol 2008;22:273-80.

24. Yantiss RK, Odze RD. Optimal approach to obtaining mucosal biopsies for assessment of inflammatory disorders of the gastrointestinal tract. Am J Gastroenterol 2009;104:774-83.

25. Hopper AD, Cross SS, Sanders DS. Patchy villous atrophy in adult patients with suspected gluten-sensitive enteropathy: Is a multiple duodenal biopsy strategy appropriate? Endoscopy 2008;40:219-24.

26. Lecleire S, Di Fiore F, Antonietti M, Savoye G, Lemoine F, Le Pessot F, et al. Endoscopic markers of villous atrophy are not useful for the detection of celiac disease in patients with dyspeptic symptoms. Endoscopy 2006;38:696-701.

27. Sonnenberg A, Lash RH, Genta RM. A national study of Helicobacter pylori infection in gastric biopsy specimens. Gastroenterology 2010;139:1894-901

28. Abrams JA, Kapel RC, Lindberg GM, Saboorian MH, Genta RM, Neugut AI, et al. Adherence to biopsy guidelines for Barrett's esophagus surveillance in the community setting in the United States. Clin Gastroenterol Hepatol 2009;7:736-42.

29. Rostami K, Villanacci V. Microscopic enteritis: novel prospect in coeliac disease clinical and immuno-histogenesis. Evolution in diagnostic and treatment strategies. Dig Liver Dis 2009;41:245-52.

30. Rodrigo-Sáez L, Fuentes-Álvarez D, Pérez-Martínez I, Álvarez-Mieres $\mathrm{N}$, Niño-García P, De-Francisco-García R, et al. Differences between pediatric and adult celiac disease. Rev Esp Enferm Dig 2011;103:238-44.

31. Rostami K, Kasturi R, Villanacci V, Bassotti G, Zambelli A. Challenges in endoscopy and histological diagnosis of celiac disease. Endoscopy 2011;43:375 